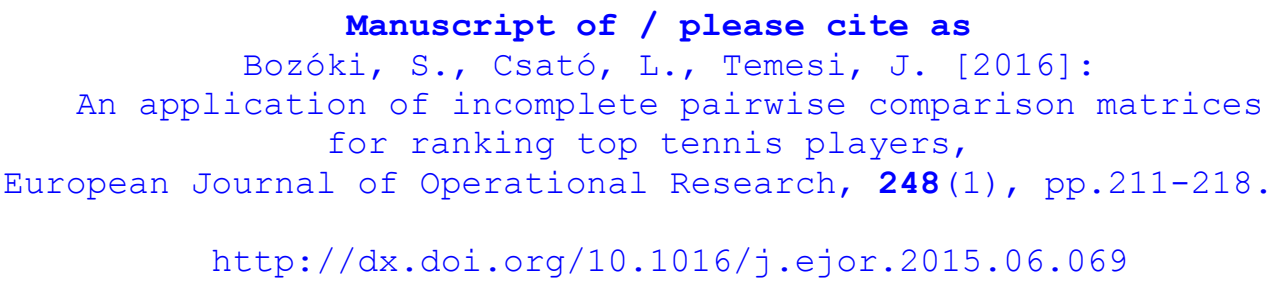

\title{
An application of incomplete pairwise comparison matrices for ranking top tennis players
}

\author{
Sándor Bozóki*- László Csató†- József Temesi ${ }^{\ddagger}$
}

November 3, 2016

\begin{abstract}
Pairwise comparison is an important tool in multi-attribute decision making. Pairwise comparison matrices (PCM) have been applied for ranking criteria and for scoring alternatives according to a given criterion. Our paper presents a special application of incomplete PCMs: ranking of professional tennis players based on their results against each other. The selected 25 players have been on the top of the ATP rankings for a shorter or longer period in the last 40 years. Some of them have never met on the court. One of the aims of the paper is to provide ranking of the selected players, however, the analysis of incomplete pairwise comparison matrices is also in the focus. The eigenvector method and the logarithmic least squares method were used to calculate weights from incomplete PCMs. In our results the top three players of four decades were Nadal, Federer and Sampras. Some questions have been raised on the properties of incomplete PCMs and remains open for further investigation.
\end{abstract}

Keywords: decision support, incomplete pairwise comparison matrix, ranking

\section{Introduction}

A well-known application field of pairwise comparison matrices (PCMs) is multi-attribute decision making (MADM). The values of pairwise comparisons are applied for ranking of criteria or for scoring alternatives to a given criterion.

This paper will use pairwise comparison values for ranking of tennis players based on their results against each other. Our aim is to make a 'historical' comparison of top tennis players of the last 40 years. The ranking idea is how the players performed against each other in a pairwise manner in the long run. We have collected the results of 25 players who have been on the top of the ATP ranking lists for a shorter or a longer period. ${ }^{1}$

${ }^{*}$ Institute for Computer Science and Control, Hungarian Academy of Sciences (MTA SZTAKI), Laboratory on Engineering and Management Intelligence, Research Group of Operations Research and Decision Systems and Department of Operations Research and Actuarial Sciences, Corvinus University of Budapest, Hungary e-mail: bozoki.sandor@sztaki.mta.hu

${ }^{\dagger}$ Department of Operations Research and Actuarial Sciences, Corvinus University of Budapest and MTA-BCE "Lendület" Strategic Interactions Research Group, Hungary

e-mail: laszlo.csato@uni-corvinus.hu

${ }^{\ddagger}$ Department of Operations Research and Actuarial Sciences, Corvinus University of Budapest, Hungary e-mail: jozsef.temesi@uni-corvinus.hu

${ }^{1}$ One can ask, why not an all-time ranking? The answer is simple: our data collection used the official ATP website. The ATP database contained reliable and complete data from 1973 (see at http://www. atpworldtour. com/Players/Head-To-Head.aspx). 
There could be several reasons why some elements of a $P C M$ are missing. It can happen that decision makers do not have time to make all comparisons, or they are not able to make some of the comparisons. Some data could have lost, but it is also possible that the comparison was not possible. In our case the reason of missing elements is obvious: we are not able to compare those players directly who have never played against each other.

Professional tennis is very popular around the world. The professional tennis associations (ATP, WTA) have been collecting data about the tournaments and the players. There is a freely available database about the results of the top tennis players including data from 1973. That gave the possibility to construct the pairwise comparison matrices of those players who have been leading the ATP ranking for a period of any length. Applying one of the estimation methods for generating a weight vector we can produce an order of the players: a ranking. That approach might be highly disputable among tennis fans, of course, but we have to note that other ranking ideas are also based on consensus or tradition, and there is no unique answer to the question 'Who is the best?'.

The existing ATP rankings, for instance, give points to the players for certain periods according to the importance of the ATP tournaments (based on the prize money) using simple rules for correcting the impacts of some biasing conditions.

The media and most of the experts consider \#1 of the ATP-ranking as the 'best' tennis player. Our approach is also ranking-oriented, but we will not use this term, the emphasis will be put on the excellence of players with higher positions relative to those who have lower ranking positions. Ranking of players will be done according to the weights, and the player with the highest weight can be regarded as the 'best', however, this term is restricted to our sample of players and varies as different ranking lists are generated.

In recent years some papers have attempted to rank professional tennis players with the use of well-founded methods. Radicchi (2011) considered all matches played between 1968 and 2010 to construct a weighted and directed preference graph. It develops a diffusion algorithm similar to Google's PageRank (Brin and Page, 1998) to derive the ranking of nodes representing the tennis players. It also provides lists for specific playing surfaces and different time periods. On the basis of the whole dataset, Jimmy Connors was identified as the \#1 player. He is also the winner of the decade 1971-80. For subsequent years, the \#1 players are Ivan Lendl (1981-1990), Pete Sampras (1991-2000) and Roger Federer (2001-2010). The new ranking has a higher predictive power than the official ATP ranking and does not require arbitrary external criteria, with the exception of a control parameter.

Dingle et al. (2013) use this method to derive PageRank-based tennis rankings instead of the official ATP and WTA rankings. For top-ranked players, they are broadly similar, but there is a wide variation in the tail. The PageRank-based rankings are found to be better predictor of match outcomes. Spanias and Knottenbelt (2013) present two new algorithms, SortRank and LadderRank, which make use of a quantitative tennis model to assess the performance of players and compare them with each other. Dahl (2012) introduce a parametric method based on linear algebra considering the importance of the matches, too. Motegi and Masuda (2012) propose a network-based dynamical ranking system, taking into account that the strength of players depend on time. The method outperforms both the official ranking and Radicchi (2011)'s prestige score in prediction accuracy.

Several authors build statistical models with the aim of a good prediction power. Clarke and Dyte (2000) argue that since the rankings are derived from a points rating, an estimate of each player's chance in a head to head contest can be made from the difference in the players' rating points. Using a year's tournament results, a logistic regression model can be fitted to the ATP ratings to estimate that chance. McHale and Morton (2011) apply a Bradley-Terry type model (Bradley and Terry, 1952) to obtain forecasts, and they show that these forecasts 
are more accurate according to several criteria than the forecasts obtained from standard models employed in the literature. They compare the model to two logit models, one using official rankings and another using the official ranking points of the two competing players. Irons et al. (2014) refine that model to be more transparent, fair and insensitive to bias. As they say, even the simplest model improves significantly over the current system, despite having three of the same constraints: no surface information is used, only match results count, and a 12 month rolling window is used to weight games.

Ruiz et al. (2013) apply Data Envelopment Analysis. According to their model, the 'efficient' players can be used for the 'inefficient' ones as benchmark in order to improve certain characteristics of their play. The ranking is based on cross-efficiency ratios.

Our paper discusses some theoretical results and applications of the incomplete pairwise comparison matrices. This section describes the aim of our research and reviews sport applications with a focus on tennis rankings. The ranking approach implies the use of pairwise comparisons in a natural way. Section 2 provides an overview of the results in the area of incomplete pairwise comparison matrices - some of them have been published previously by the authors of this paper. The applied model for top professional tennis players is introduced in the first part of Section 3 together with the description of the database and methodology. The second part of Section 3 describes the derived rankings - the Eigenvector Method and the Logarithmic Least Squares Method are applied -, and analyzes some properties of these results. Section 4 includes further analysis and draws conclusions with some remaining open questions.

\section{Theory and methods}

Our paper applies the method of pairwise comparisons.

Definition 2.1. Pairwise comparison matrix: Let $\mathbb{R}_{+}^{n \times n}$ denote the class of $n \times n$ matrices with positive real elements. The matrix

$$
\mathbf{A}=\left(\begin{array}{ccccc}
1 & a_{12} & a_{13} & \ldots & a_{1 n} \\
1 / a_{12} & 1 & a_{23} & \ldots & a_{2 n} \\
1 / a_{13} & 1 / a_{23} & 1 & \ldots & a_{3 n} \\
\vdots & \vdots & \vdots & \ddots & \vdots \\
1 / a_{1 n} & 1 / a_{2 n} & 1 / a_{3 n} & \ldots & 1
\end{array}\right) \in \mathbb{R}_{+}^{n \times n}
$$

is called a pairwise comparison matrix, if

$$
a_{i i}=1 \quad \text { and } \quad a_{i j}=\frac{1}{a_{j i}}
$$

for all indices $i, j=1, \ldots, n$.

In our case the alternatives are tennis players. Choosing any two of them $\left(P_{i}\right.$ and $\left.P_{j}\right)$, we have the results of all matches have been played between them. Let the number of winning matches of $P_{i}$ over $P_{j}$ be $x$, and the number of lost matches $y$. We can construct the ratio $x_{i} / y_{i}$ : if it is greater than 1 , we can say that $P_{i}$ is a 'better' player than $P_{j}$. In case of $x_{i} / y_{i}$ is equal to 1 we are not able to decide who is the better. Let the $a_{i j}$ element of the matrix $A$ be $x_{i} / y_{i}$, and the $a_{j i}$ element be $y_{i} / x_{i}$ for all $i, j=1, \ldots, n, i \neq j$. Choose the diagonal elements $a_{i i}=1$ for all $i=1,2, \ldots, n$, thus $\mathbf{A}$ becomes a pairwise comparison matrix according to Definition 2.1.

The PCM matrix $\mathbf{A}$ is used to determine a weight vector $\mathbf{w}=\left(w_{1}, w_{2}, \ldots, w_{n}\right), w_{i}>0,(i=$ $1, \ldots, n)$, where the elements $a_{i j}$ are estimated by $w_{i} / w_{j}$. Since the estimated values are ratios, 
it is a usual normalization condition that the sum of the weights is equal to $1: \sum_{i=1}^{n} w_{i}=1$. That estimation problem can be formulated in several ways. Saaty (Saaty, 1980) formulated an eigenvalue problem in the Analytic Hierarchy Process $(A H P)$, where the components of the right eigenvector belonging to the maximal eigenvalue $\left(\lambda_{\max }\right)$ of matrix $\mathbf{A}$ will give the weights. We will refer to that procedure as the Eigenvector Method $(E M)$.

For solving the estimation problem it could be obvious to apply methods based on distance minimization, too. That approach will estimate the elements of the $\mathbf{A}$ matrix with the elements of a matrix $\mathbf{W}$, where the element $w_{i j}$ of $\mathbf{W}$ is $w_{i} / w_{j}, w_{i}$ and $w_{j}>0,(i, j=1, \ldots, n)$, and the objective function to be minimized is the distance of the two matrices. Choo and Wedley (2004) categorized the estimation methods and found 12 different distance minimization methods of deriving $\mathbf{w}$ from $\mathbf{A}$ based on minimizing the absolute deviation $\left|a_{i j}-w_{i} / w_{j}\right|$ or $\left|w_{j} a_{i j}-w_{i}\right|$, or minimizing the square $\left(a_{i j}-w_{i} / w_{j}\right)^{2}$ or $\left(w_{j} a_{i j}-w_{i}\right)^{2}$. The effectiveness of some methods has been studied by Lin (2007). We will use the Logarithmic Least Squares Method (LLSM) (Crawford and Williams, 1985; De Graan, 1980; Rabinowitz, 1976).

Several authors deal with the problem of inconsistency in AHP (see e.g. Bana e Costa and Vansnick (2008)). In our tennis application intransitivity may occur, therefore inconsistency is a natural phenomenon. However, the data set is given, consistency correction of the matrix elements could not be done.

PCMs may be incomplete, that is, they have missing entries denoted by $*$ :

$$
\mathbf{A}=\left(\begin{array}{ccccc}
1 & a_{12} & * & \ldots & a_{1 n} \\
1 / a_{12} & 1 & a_{23} & \ldots & * \\
* & 1 / a_{23} & 1 & \ldots & a_{3 n} \\
\vdots & \vdots & \vdots & \ddots & \vdots \\
1 / a_{1 n} & * & 1 / a_{3 n} & \ldots & 1
\end{array}\right)
$$

Main results have been discussed by Harker (Harker, 1987), Carmone, Kara and Zanakis (Carmone et al., 1997), Kwiesielewicz and van Uden (Kwiesielewicz, 1996; Kwiesielewicz and van Uden, 2003), Shiraishi, Obata and Daigo (Shiraishi et al., 1998; Shiraishi and Obata, 2002), Takeda and Yu (Takeda and Yu, 1995), Fedrizzi and Giove (Fedrizzi and Giove, 2007).

Definition 2.2. Graph representation of a PCM: Undirected graph $G:=(V, E)$ represents the incomplete pairwise comparison matrix $\mathbf{A}$ of size $n \times n$ such that $V=\{1,2, \ldots, n\}$ the vertices correspond to the objects to compare and $E=\left\{e(i, j) \mid a_{i j}\right.$ is given and $\left.i \neq j\right\}$, that is, the edges correspond to the known matrix elements.

There are no edges corresponding to the missing elements in the matrix.

Kwiesielewicz (Kwiesielewicz, 1996) have considered the Logarithmic Least Squares Method $(L L S M)$ for incomplete matrices as

$$
\begin{aligned}
\min \sum_{(i, j): a_{i j} \text { is given }}\left[\log a_{i j}-\log \left(\frac{w_{i}}{w_{j}}\right)\right]^{2} \\
\sum_{i=1}^{n} w_{i}=1, \\
w_{i}>0, \quad i=1,2, \ldots, n .
\end{aligned}
$$

Theorem 2.1. (Bozóki et al., 2010, Theorem 4) Optimization problem (2)-(4) has a unique solution if and only if $\mathrm{G}$ is connected. Furthermore, the optimal solution is calculated by solving a system of linear equations. 
Note that the incomplete $L L S M$ problem asks for the weights, however, missing elements can be calculated as the ratio of the corresponding optimal weights. We will focus only on the weights.

The generalization of the eigenvector method to the incomplete case requires two steps. First, positive variables $x_{1}, x_{2}, \ldots, x_{d}$ are written instead of missing elements as follows:

$$
\mathbf{A}(\mathbf{x})=\mathbf{A}\left(x_{1}, x_{2}, \ldots, x_{d}\right)=\left(\begin{array}{ccccc}
1 & a_{12} & x_{1} & \ldots & a_{1 n} \\
1 / a_{12} & 1 & a_{23} & \ldots & x_{d} \\
1 / x_{1} & 1 / a_{23} & 1 & \ldots & a_{3 n} \\
\vdots & \vdots & \vdots & \ddots & \vdots \\
1 / a_{1 n} & 1 / x_{d} & 1 / a_{3 n} & \ldots & 1
\end{array}\right)
$$

Let $\mathbf{x}=\left(x_{1}, x_{2}, \ldots, x_{d}\right)^{T} \in \mathbb{R}_{+}^{d}$.

Saaty (Saaty, 1980) defined inconsistency index $C R$ as a positive linear transformation of $\lambda_{\max }(\mathbf{A})$ such that $C R(\mathbf{A}) \geq 0$ and $C R(\mathbf{A})=0$ if and only if $\mathbf{A}$ is consistent. The idea that larger $\lambda_{\text {max }}$ indicates higher $C R$ inconsistency led Shiraishi, Obata and Daigo (Shiraishi et al., 1998; Shiraishi and Obata, 2002) to consider the eigenvalue optimization problem

$$
\min _{\mathbf{x}>\mathbf{0}} \lambda_{\max }(\mathbf{A}(\mathbf{x}))
$$

in order to find a completion that minimizes the maximal eigenvalue, or, equivalently, $C R$. As in case of incomplete $L L S M$, uniqueness is closely related to the connectedness of $G$.

Theorem 2.2. (Bozóki et al., 2010, Theorem 2, Corollary 2 and Section 5) Optimization problem (6) has a unique solution if and only if $G$ is connected. Furthermore, (6) can be transformed to a convex optimization problem that can be solved efficiently.

Second step is to apply the eigenvector method to the completed pairwise comparison matrix.

Parallel with publishing the first theoretical results on incomplete $P C M$ s our research team have been seeking for applications. The world of sports provided us a prosperous experimental field. Csató (2013) has analysed the chess olympiad. A research paper was published later as a chapter in a book in Hungarian (Temesi et al., 2012) on ranking tennis players. Some early results have been published and some research questions have been formulated there. The recent article expands the scope of the research and reports new results.

\section{Calculation and results}

Our aim is to demonstrate that it is possible to compare players from a long period of time. There are several options how to choose from the list of professional players included in the ATP database. All choices have pros and cons. There is no ideal set of players and comparative time periods, because experts have disputes on controversial issues: Who can represent a certain era? May we compare results from different periods of the carrier path of an individual player? May we set up a unified ranking or it is better to have separate rankings for different surfaces?

We have chosen those 25 players who have been \#1 on the ATP ranking for any period of time from 1973. Figure 1 shows them together with their active period in the world of professional tennis.

In our calculations the initial data are as follows:

- $z_{i j}(i, j=1, \ldots, n, i \neq j)$ : the number of matches have been played between players $P_{i}$ and $P_{j}\left(z_{i j}=z_{j i}\right)$ 
Figure 1: Length of professional tennis career for the chosen players

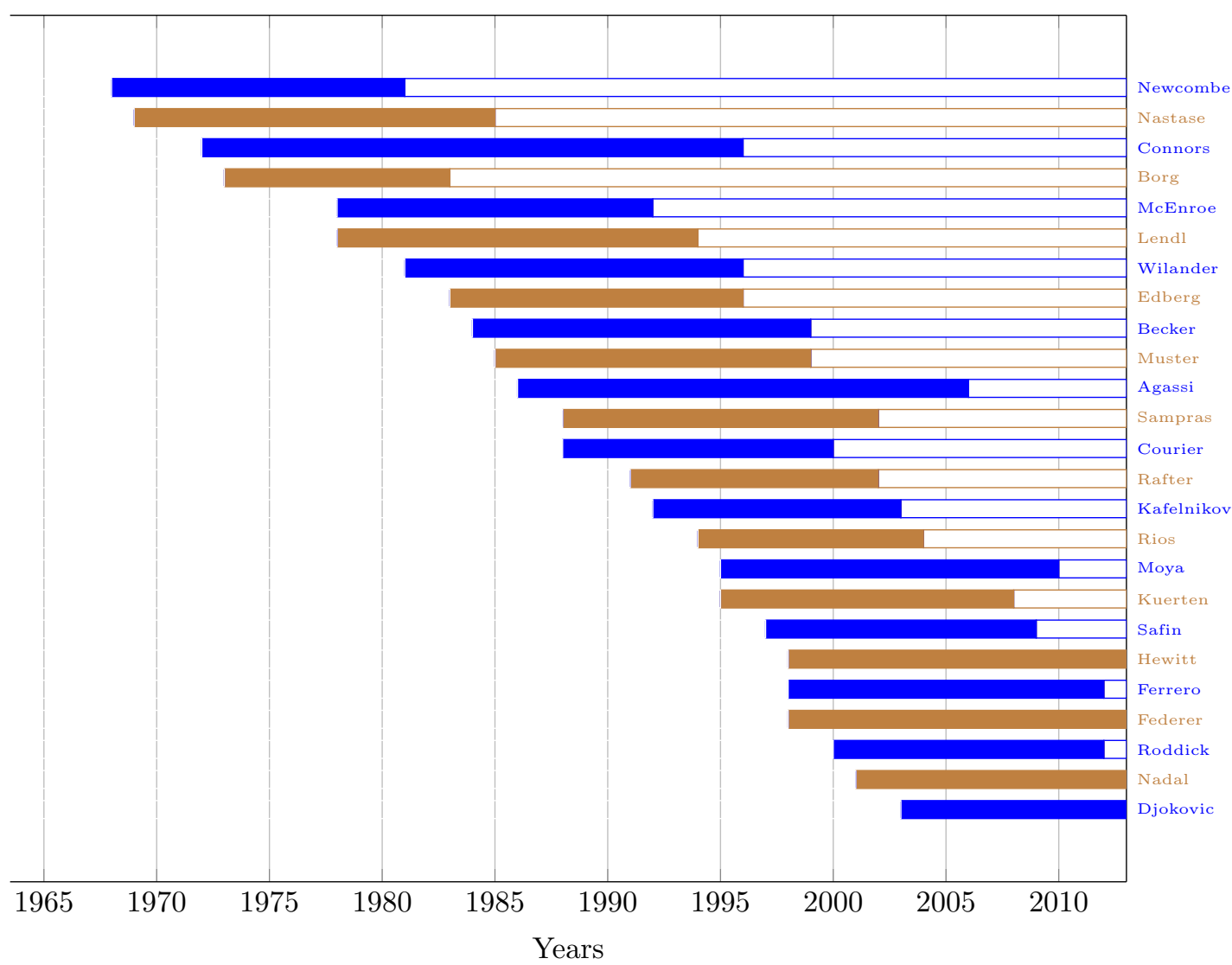

- $x_{i j}(i>j)$ : the number of matches between players $P_{i}$ and $P_{j}$, where $P_{i}$ was the winner;

- $y_{i j}=z_{i j}-x_{i j}(i>j)$ : the number of matches between players $P_{i}$ and $P_{j}$, where $P_{i}$ lost against $P_{j}$.

Definition 3.1. Pairwise comparison matrix of top tennis players: $p_{i j}$ elements of matrix $P$ are calculated from the initial data as

- $p_{i j}=x_{i j} / y_{i j}$ if $i, j=1, \ldots, n, i>j$ and $x_{i j} \neq 0, y_{i j} \neq 0$;

- $p_{j i}=y_{i j} / x_{i j}=1 / p_{i j}$ if $i, j=1, \ldots, n, i<j$ and $x_{i j} \neq 0, z_{i j} \neq 0$;

- $p_{i i}=1$ for all $i=1, \ldots, n$;

- $p_{i j}$ and $p_{j i}$ elements are missing otherwise.

A consequence of the definition is that in case of $z_{i j}=0$ for at least one pair of the players, the pairwise comparison matrix is incomplete. The interpretation of $p_{i j}>0$ is that the $i$ th player is $p_{i j}$ times better than the $j$ th player.

We have to note that Definition 3.1 is strict in the sense that $p_{i j}$ is also missing in the case when $z_{i j} \neq 0$, but one of its component is 0 (either $x_{i j}=0$ or $y_{i j}=0$ ). However, it can happen 
that $P_{i}$ won several times over $P_{j}$, and he has never been defeated. According to the definition we can eliminate all pairs where that phenomenon occurs, but it would be unfair for the winner player in the given pair. Therefore we decided to use artificial $p_{i j}$ values for these cases. For instance $p_{i j}=5$ was used if $z_{i j}$ was less than 5 and $y_{i j}=0, p_{i j}=10$ if the number of matches was between 6 and 10 , and $y_{i j}=0$, and so on. In our calculations we will use this correction method and we will refer to it with a subscript 1 . Another correction method for $p_{i j}$ could be that the value of $p_{i j}=x_{i j}+2$ if $y_{i j}=0$. In our calculations we will refer to that correction method with a subscript 2 (see Table 2 and 3 later).

One can naturally argue that the choice of $p_{i j}$ is crucial to get different results. We have made a series of calculations for various numbers of players with several correction values (Temesi et al., 2012) and we have found that the results did not alter significantly.

Table 1 contains the results of the matches played between players $P_{i}$ and $P_{j}$ (sum of the symmetric elements of the matrix is $z_{i j}$ for all players). We can see that there are cases when two other players have played more than 30 times with each other, and it was also possible that two players met less than 5 times. There is a need for balancing the impact of extremely differing match numbers resulted in a wide range of ratios. In order to handle that problem we introduced a transformation for the elements of $p_{i j}$ :

$$
t_{i j}=p_{i j}^{z_{i j} / \max z_{i j}}
$$

where the transforming factor is the ratio of the number of matches between each other divided by the maximum number of matches of all pairs.

Note that if all players have the same number of matches, transformation (7) results in $t_{i j}=p_{i j}$. It approximates 1 when the two players have played a small number of matches against each other, therefore the outcome seems to be 'unreliable'. For instance, the original $p_{i j}$ value for the pair Agassi-Becker was $10 / 4=2.5$, the transformed $t_{i j}$ value is $(10 / 4)^{14 / 39}=1.3895$ where 14 is the number of matches between Agassi and Becker, and 39 is the maximum of $z_{i j}$ values (Djokovic vs. Nadal).

The vertices of the graph in Figure 2 represent the players. The edges show that the two players played at least one match against each other. The bold lines connected to the node labelled Agassi illustrate that he played against 20 of our players during his carrier: the degree of the vertex is 20 (which is also the maximum degree). Edges from Agassi to other players (e.g. to Connors) mean that Agassi has more wins than losses against them (indicated also in the neighbouring table). Similarly, edges to Agassi from other players (e.g. from Rios) mean that Agassi has more losses than wins against them, while dashed lines represent an equal number of wins and losses (e.g. to Safin). We have plotted the graphs belonging to all players in an Online Appendix, available at http://www.sztaki.mta.hu/ bozoki/tennis/appendix.pdf.

Having the incomplete pairwise comparison matrices for the 25 top tennis players from Table 1 we can calculate the weight vectors if the corresponding matrix $\mathbf{T}$ is connected. It can be checked that this condition is met: the 20 edges adjacent to the node Agassi together with the edges Nadal-Djokovic, Newcombe-Nastase, Nastase-Connors and Connors-Borg form a spanning tree (see Figure 2). 
Table 1: Pairwise comparisons: number of wins/total number of matches

\begin{tabular}{|c|c|c|c|c|c|c|c|c|c|c|c|c|c|c|c|c|c|c|c|c|c|c|c|c|c|c|}
\hline & 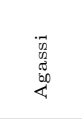 & 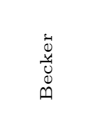 & $\begin{array}{l}\text { on } \\
\stackrel{0}{0}\end{array}$ & $\begin{array}{l}\text { ñ } \\
0 \\
0 \\
0 \\
0 \\
0\end{array}$ & 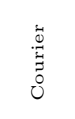 & $\begin{array}{l}0 \\
\stackrel{0}{0} \\
\frac{0}{0} \\
\stackrel{0}{0}\end{array}$ & 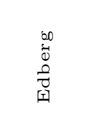 & 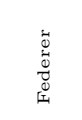 & 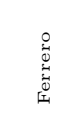 & 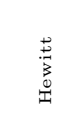 & 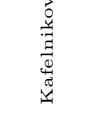 & 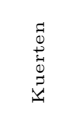 & $\begin{array}{l}\overrightarrow{\tilde{J}} \\
\stackrel{\Xi}{9}\end{array}$ & 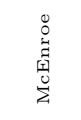 & 离 & $\begin{array}{l}\text { 蒂 } \\
\text { 咅 }\end{array}$ & $\begin{array}{l}\overrightarrow{\widetilde{J}} \\
\tilde{\tilde{\sigma}} \\
\bar{z}\end{array}$ & 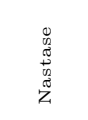 & 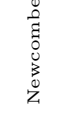 & 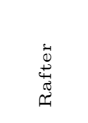 & $\stackrel{n}{\stackrel{n}{*}}$ & 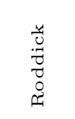 & 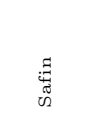 & 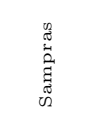 & 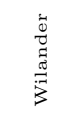 & Total \\
\hline Agassi & & $10 / 14$ & & $2 / 2$ & $5 / 12$ & & $6 / 9$ & $3 / 11$ & $2 / 5$ & $4 / 8$ & $8 / 12$ & $7 / 11$ & $2 / 8$ & $2 / 4$ & $3 / 4$ & $5 / 9$ & $0 / 2$ & & & $10 / 15$ & $1 / 3$ & $5 / 6$ & $3 / 6$ & $14 / 34$ & $5 / 7$ & $97 / 182$ \\
\hline Becker & $4 / 14$ & & & $6 / 6$ & $6 / 7$ & & $25 / 35$ & & & $1 / 1$ & $4 / 6$ & & $10 / 21$ & $8 / 10$ & $2 / 4$ & $2 / 3$ & & $1 / 1$ & & $2 / 3$ & $3 / 5$ & & $0 / 1$ & $7 / 19$ & $7 / 10$ & $88 / 146$ \\
\hline Borg & & & & $15 / 23$ & & & & & & & & & $6 / 8$ & $7 / 14$ & & & & $10 / 15$ & $1 / 4$ & & & & & & $1 / 1$ & $40 / 65$ \\
\hline Connors & $0 / 2$ & $0 / 6$ & $8 / 23$ & . & $0 / 3$ & & $6 / 12$ & & & & & & $13 / 34$ & $14 / 34$ & & & & $12 / 27$ & $2 / 4$ & & & & & $0 / 2$ & $0 / 5$ & $55 / 152$ \\
\hline Courier & $7 / 12$ & $1 / 7$ & & $3 / 3$ & - & & $6 / 10$ & & & & $1 / 6$ & $1 / 1$ & $0 / 4$ & $2 / 3$ & $2 / 3$ & $7 / 12$ & & $0 / 1$ & & $0 / 3$ & $0 / 3$ & & $1 / 2$ & $4 / 20$ & & $35 / 90$ \\
\hline Djokovic & & & & & & & & $15 / 31$ & $2 / 3$ & $6 / 7$ & & & & & $2 / 4$ & & $17 / 39$ & & & & & $4 / 9$ & $0 / 2$ & & & $46 / 95$ \\
\hline Edberg & $3 / 9$ & $10 / 35$ & & $6 / 12$ & $4 / 10$ & & & & & & $1 / 3$ & & $14 / 27$ & $6 / 13$ & $1 / 1$ & $10 / 10$ & & & & $3 / 3$ & $1 / 1$ & & & $6 / 14$ & $9 / 20$ & $74 / 158$ \\
\hline Federer & $8 / 11$ & & & & & $16 / 31$ & & & $10 / 13$ & $18 / 26$ & $2 / 6$ & $1 / 3$ & & & $7 / 7$ & & $10 / 32$ & & & $0 / 3$ & $2 / 2$ & $21 / 24$ & $10 / 12$ & $1 / 1$ & & $106 / 171$ \\
\hline Ferrero & $3 / 5$ & & & & & $1 / 3$ & & $3 / 13$ & 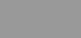 & $4 / 10$ & $1 / 3$ & $3 / 5$ & & & $8 / 14$ & & $2 / 9$ & & & $2 / 3$ & $3 / 4$ & $0 / 5$ & $6 / 12$ & & & $36 / 86$ \\
\hline Hewitt & $4 / 8$ & $0 / 1$ & & & & $1 / 7$ & & $8 / 26$ & $6 / 10$ & 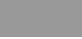 & $7 / 8$ & $3 / 4$ & & & $7 / 12$ & & $4 / 10$ & & & $3 / 4$ & $3 / 5$ & $7 / 14$ & $7 / 14$ & $5 / 9$ & & $65 / 132$ \\
\hline Kafelnikov & $4 / 12$ & $2 / 6$ & & & $5 / 6$ & & $2 / 3$ & $4 / 6$ & $2 / 3$ & $1 / 8$ & 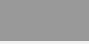 & $5 / 12$ & & & $3 / 6$ & $1 / 5$ & & & & $3 / 5$ & $6 / 8$ & & $2 / 4$ & $2 / 13$ & $1 / 2$ & $43 / 99$ \\
\hline Kuerten & $4 / 11$ & & & & $0 / 1$ & & & $2 / 3$ & $2 / 5$ & $1 / 4$ & $7 / 12$ & 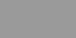 & & & $4 / 7$ & $3 / 3$ & & & & $4 / 8$ & $2 / 4$ & $1 / 2$ & $4 / 7$ & $1 / 3$ & & $35 / 70$ \\
\hline Lendl & $6 / 8$ & $11 / 21$ & $2 / 8$ & $21 / 34$ & $4 / 4$ & & $13 / 27$ & & & & & & & $21 / 36$ & & $4 / 5$ & & $1 / 1$ & & $0 / 1$ & & & & $3 / 8$ & $15 / 22$ & $101 / 175$ \\
\hline McEnroe & $2 / 4$ & $2 / 10$ & $7 / 14$ & $20 / 34$ & $1 / 3$ & & $7 / 13$ & & & & & & $15 / 36$ & & & & & $6 / 9$ & $1 / 2$ & & & & & $0 / 3$ & $7 / 13$ & $68 / 141$ \\
\hline Moya & $1 / 4$ & $2 / 4$ & & & $1 / 3$ & $2 / 4$ & $0 / 1$ & $0 / 7$ & $6 / 14$ & $5 / 12$ & $3 / 6$ & $3 / 7$ & & & . & $4 / 8$ & $2 / 8$ & & & $3 / 4$ & $2 / 7$ & $1 / 5$ & $4 / 7$ & $1 / 4$ & & $40 / 105$ \\
\hline Muster & $4 / 9$ & $1 / 3$ & & & $5 / 12$ & & $0 / 10$ & & & & $4 / 5$ & $0 / 3$ & $1 / 5$ & & $4 / 8$ & & & & & $0 / 3$ & $3 / 4$ & & $0 / 1$ & $2 / 11$ & $0 / 2$ & $24 / 76$ \\
\hline Nadal & $2 / 2$ & & & & & $22 / 39$ & & $22 / 32$ & $7 / 9$ & $6 / 10$ & & & & & $6 / 8$ & & & & & & & $7 / 10$ & $2 / 2$ & & & $74 / 112$ \\
\hline Nastase & & $0 / 1$ & $5 / 15$ & $15 / 27$ & $1 / 1$ & & & & & & & & $0 / 1$ & $3 / 9$ & & & & & $4 / 5$ & & & & & & $0 / 1$ & $28 / 60$ \\
\hline Newcombe & & & $3 / 4$ & $2 / 4$ & & & & & & & & & & $1 / 2$ & & & & $1 / 5$ & & & & & & & & $7 / 15$ \\
\hline Rafter & $5 / 15$ & $1 / 3$ & & & $3 / 3$ & & $0 / 3$ & $3 / 3$ & $1 / 3$ & $1 / 4$ & $2 / 5$ & $4 / 8$ & $1 / 1$ & & $1 / 4$ & $3 / 3$ & & & & . & $2 / 3$ & & $1 / 1$ & $4 / 16$ & $1 / 3$ & $33 / 78$ \\
\hline Rios & $2 / 3$ & $2 / 5$ & & & $3 / 3$ & & $0 / 1$ & $0 / 2$ & $1 / 4$ & $2 / 5$ & $2 / 8$ & $2 / 4$ & & & $5 / 7$ & $1 / 4$ & & & & $1 / 3$ & 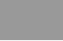 & $0 / 2$ & $1 / 4$ & $0 / 2$ & & $22 / 57$ \\
\hline Roddick & $1 / 6$ & & & & & $5 / 9$ & & $3 / 24$ & $5 / 5$ & $7 / 14$ & & $1 / 2$ & & & $4 / 5$ & & $3 / 10$ & & & & $2 / 2$ & & $4 / 7$ & $2 / 3$ & & $37 / 87$ \\
\hline Safin & $3 / 6$ & $1 / 1$ & & & $1 / 2$ & $2 / 2$ & & $2 / 12$ & $6 / 12$ & $7 / 14$ & $2 / 4$ & $3 / 7$ & & & $3 / 7$ & $1 / 1$ & $0 / 2$ & & & $0 / 1$ & $3 / 4$ & $3 / 7$ & 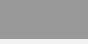 & $4 / 7$ & & $41 / 89$ \\
\hline Sampras & $20 / 34$ & $12 / 19$ & & $2 / 2$ & $16 / 20$ & & $8 / 14$ & $0 / 1$ & & $4 / 9$ & $11 / 13$ & $2 / 3$ & $5 / 8$ & $3 / 3$ & $3 / 4$ & $9 / 11$ & & & & $12 / 16$ & $2 / 2$ & $1 / 3$ & $3 / 7$ & & $2 / 3$ & $115 / 172$ \\
\hline Wilander & $2 / 7$ & $3 / 10$ & $0 / 1$ & $5 / 5$ & & & $11 / 20$ & & & & $1 / 2$ & & $7 / 22$ & $6 / 13$ & & $2 / 2$ & & $1 / 1$ & & $2 / 3$ & & & & $1 / 3$ & & $41 / 89$ \\
\hline
\end{tabular}


Figure 2: Graph representation of matrix $\mathbf{T}$

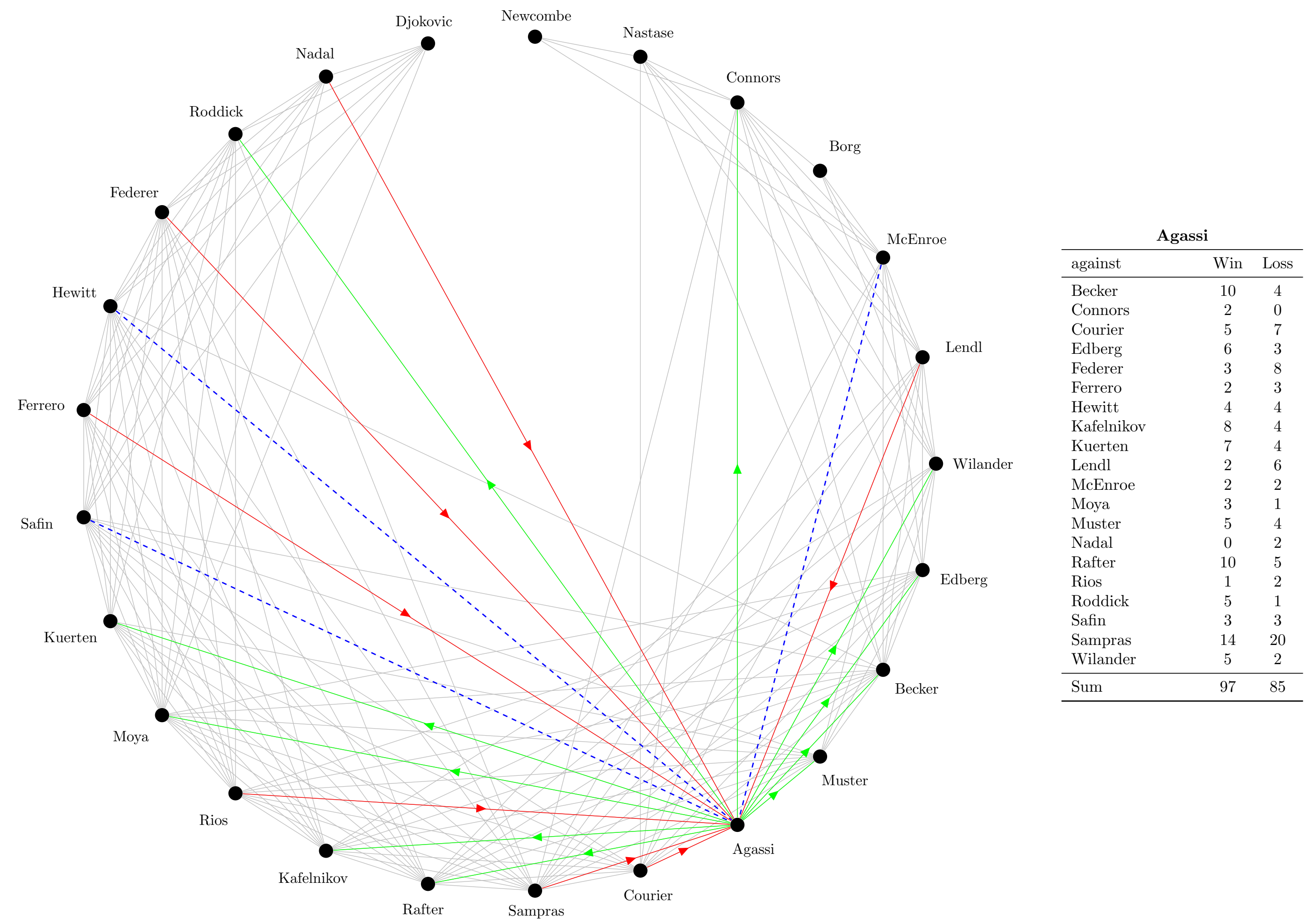


Table 2: Rankings

\begin{tabular}{|c|c|c|c|c|c|}
\hline & $E M_{2}$ & $L L S M_{2}$ & $E M_{W 2}$ & $L L S M_{W 2}$ & $\mathrm{~W} / \mathrm{L}$ \\
\hline Nadal & 1 & 1 & 1 & 1 & 2 \\
\hline Federer & 2 & 2 & 2 & 2 & 3 \\
\hline Sampras & 3 & 3 & 3 & 3 & 1 \\
\hline Lendl & 11 & 8 & 4 & 4 & 6 \\
\hline Borg & 13 & 11 & 6 & 5 & 4 \\
\hline Becker & 4 & 4 & 5 & 6 & 5 \\
\hline Djokovic & 5 & 5 & 7 & 7 & 10 \\
\hline Agassi & 9 & 9 & 8 & 8 & 7 \\
\hline Hewitt & 6 & 7 & 9 & 9 & 9 \\
\hline Kuerten & 16 & 15 & 10 & 10 & 8 \\
\hline Safin & 12 & 10 & 11 & 11 & 15 \\
\hline McEnroe & 20 & 18 & 12 & 12 & 11 \\
\hline Nastase & 22 & 20 & 14 & 13 & 13 \\
\hline Ferrero & 17 & 16 & 16 & 14 & 20 \\
\hline Roddick & 8 & 6 & 13 & 15 & 18 \\
\hline Wilander & 15 & 14 & 17 & 16 & 16 \\
\hline Rios & 21 & 22 & 18 & 17 & 22 \\
\hline Rafter & 7 & 13 & 15 & 18 & 19 \\
\hline Newcombe & 23 & 21 & 21 & 19 & 14 \\
\hline Kafelnikov & 14 & 17 & 19 & 20 & 17 \\
\hline Moya & 19 & 19 & 22 & 21 & 23 \\
\hline Edberg & 10 & 12 & 20 & 22 & 12 \\
\hline Courier & 18 & 23 & 23 & 23 & 21 \\
\hline Muster & 24 & 24 & 24 & 24 & 25 \\
\hline Connors & 25 & 25 & 25 & 25 & 24 \\
\hline
\end{tabular}

Weight vectors have been computed with the Logarithmic Least Squares Method ( $L L S M$ in Table 2) and with the Eigenvector Method $(E M)$ as it was described in Section 2. On the basis of the weight vectors, eight rankings have been calculated without and with transformation (in the latter case we used the subscript $W$ for identification), and different correction methods have also been applied (subscripts 1 and 2 as it was introduced earlier). Selected results are demonstrated in Table 2. The fourth column, $L L S M_{W 2}$, for example, is a ranking given by Logarithmic Least Squares Method with the second correction procedure and transformed data. Note that the players are listed in Table 2 according to this ranking. The fifth column includes the ranking according to the win to loss ratio, indicated by $\mathrm{W} / \mathrm{L}$.

Rankings were practically the same with both estimation methods, as it can be seen from Table 2. The impact of the correction method is not significant either. (That was the reason why Table 2 does not contains calculations with the first type correction.) The values of the Spearman rank correlation coefficients in Table 3 support these propositions: the elements of the top-left and bottom-right $4 \times 4$ submatrices are close to the identity matrix. The correlation coefficients - comparing rankings with the same estimation method - suggest that filtering the impact of differences in the total match numbers eliminated the minor impact of the correction methods, too. Analysing the impact of the estimation methods and various forms of data correction the authors had similar experience with 34 top players (Temesi et al., 2012).

However, data transformation (7) may change the rankings significantly, as it can be seen in 
Table 3: Spearman rank correlation coefficients

\begin{tabular}{lcccccccc}
\hline & $E M_{1}$ & $E M_{2}$ & $L L S M_{1}$ & $L L S M_{2}$ & $E M_{W 1}$ & $E M_{W 2}$ & $L L S M_{W 1}$ & $L L S M_{W 2}$ \\
\hline$E M_{1}$ & 1 & 0.9715 & 0.9269 & 0.9154 & 0.7546 & 0.7423 & 0.6869 & 0.6631 \\
$E M_{2}$ & 0.9715 & 1 & 0.9677 & 0.9569 & 0.8015 & 0.7908 & 0.7385 & 0.7177 \\
$L L S M_{1}$ & 0.9269 & 0.9677 & 1 & 0.9915 & 0.8638 & 0.8469 & 0.8085 & 0.7946 \\
$L L S M_{2}$ & 0.9154 & 0.9569 & 0.9915 & 1 & 0.8931 & 0.8831 & 0.8446 & 0.8338 \\
$E M_{W 1}$ & 0.7546 & 0.8015 & 0.8638 & 0.8931 & 1 & 0.9962 & 0.9908 & 0.9854 \\
$E M_{W 2}$ & 0.7423 & 0.7908 & 0.8469 & 0.8831 & 0.9962 & 1 & 0.9900 & 0.9877 \\
$L L S M_{W 1}$ & 0.6869 & 0.7385 & 0.8085 & 0.8446 & 0.9908 & 0.9900 & 1 & 0.9969 \\
$L L S M_{W 2}$ & 0.6631 & 0.7177 & 0.7946 & 0.8338 & 0.9854 & 0.9877 & 0.9969 & 1 \\
\hline
\end{tabular}

Table 3, too. The corresponding rank correlation coefficients in the top-right and bottom-left $4 \times 4$ submatrices confirm this statement. According to our interpretation the value judgement of the ranking expert determines the choice between these rankings. Therefore if the expert's opinion is that a ratio of 2 has to be represented in different ways if it was resulted from 6 matches $(4: 2)$ or from 30 matches $(20: 10)$ than the recommended normalization has to be implemented and the corresponding ranking can be chosen.

Turning back to Table 2, the first three players (Nadal, Federer, Sampras) and the last three players (Courier, Muster, Connors) are the same in both rankings. Some differences in the rank numbers can be found in other parts of the list. Tennis fans can debate the final ranking, of course. One can compare these rankings to the win /loss ratio of the players, given in the ninth column of Table 2. However, the most important fact is that the Top 12 includes big names from the recent championships and from the good old times, as well. The conclusion is that it is possible to produce rankings based on pairwise comparisons and overarching four decades with players who have never met on the court.

\section{Conclusions and open questions}

In case of having historical data incomplete pairwise comparison matrices can be applied in order to answer the question: what is the ranking of the players for a long time period? Who is the \#1 player? With this methodology it is possible to use face to face match results. Various types of transformations can modify the original data set with the intention of correcting either data problems or biasing factors. However, we did not take into account the impact of the carrier path of a player. Every match had identical weight without considering its position on the time line. Different surfaces did not play specific role, either.

Having had a great number of calculations with tennis results we have been interested in finding answer for the question 'What are those properties of matrix $\mathbf{T}$ which have an impact on the ranking?'

Ranking can depend on the number and the distribution of the comparisons. The number of comparisons can be characterized by the density (sparsity) of the PCM. For a fully completed PCM the density of the matrix is 1 . Lower values mean that the matrix is incomplete. In our case the density of $\mathbf{T}$ is $341 / 625 \approx 0.5456$ since 341 elements are known in the $25 \times 25$ matrix.

Another indicator of the structure of matrix $\mathbf{T}$ is the distribution of elements, which can be characterized by the degree of vertices in the graph representation of the PCM. We have designed a tool for exploring the connectedness of the incomplete pairwise comparison matrices visually. Figure 3 shows the distribution of degrees in our case, which can be checked in the Online Appendix (http://www.sztaki.mta.hu/ bozoki/tennis/appendix.pdf), where clicking on a 
node shows the edges adjacent to it. The maximum degree is 20 in the case of Agassi.

Figure 3: Degree of vertices in the graph representation of matrix $\mathbf{T}$

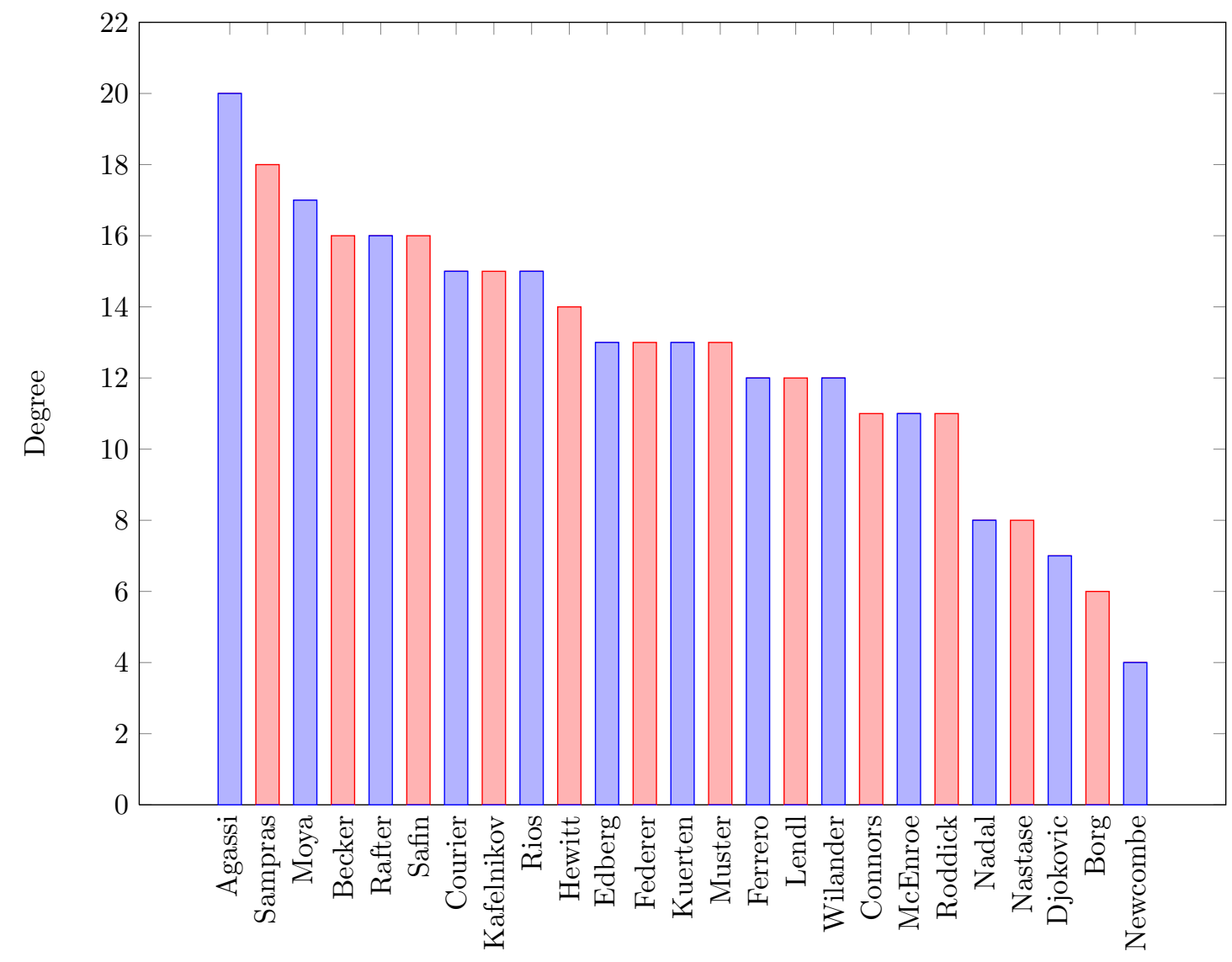

Moreover, increasing the number of matches played between those who have been played with each other leaves the value of density and the degree of vertices unchanged but the ranking can change as a result of the estimation method. An interesting question is 'How an additional match with a given result affects the ranking?' The impact of lower and higher values of sparsity (degrees of vertices) can also be analysed. A challenging question could be 'Which player can be cancelled without changing the ranking?'

The inconsistency of pairwise comparison matrices plays an important role both in theory and practice (Kéri, 2011). Further research includes the analysis of inconsistency of incomplete pairwise comparison matrices of large size. In our case we cannot speak of the inconsistency of a decision maker since the matrix elements originate from tennis matches, we might also say: from life. Intransitive triads ( $A$ beats $B, B$ beats $C$, and $C$ beats $A$ ) occur often in sports. We have found 50 intransitive triads in our example, they are plotted in the Online Appendix, available at http://www.sztaki.mta.hu/ bozoki/tennis/appendix.pdf. We hope to return to the problem of analysing intransitive triads in a(n incomplete) pairwise comparison matrix, or, equivalently, in the directed graph associated. 


\section{Acknowledgement}

The authors are grateful to the anonymous reviewers for valuable and constructive recommendations. Research was supported in part by OTKA grants K 77420 and K 111797.

\section{References}

Bana e Costa, C. A. and Vansnick, J.-C. (2008). A critical analysis of the eigenvalue method used to derive priorities in AHP. European Journal of Operational Research, 187(3):1422-1428.

Bozóki, S., Fülöp, J., and Rónyai, L. (2010). On optimal completion of incomplete pairwise comparison matrices. Mathematical and Computer Modelling, 52(1-2):318-333.

Bradley, R. A. and Terry, M. E. (1952). Rank analysis of incomplete block designs: I. The method of paired comparisons. Biometrika, 39(3/4):324-345.

Brin, S. and Page, L. (1998). The anatomy of a large-scale hypertextual web search engine. Computer networks and ISDN systems, 30(1):107-117.

Carmone, F., Kara, A., and Zanakis, S. H. (1997). A Monte Carlo investigation of incomplete pairwise comparison matrices in AHP. European Journal of Operational Research, 102(3):538553.

Choo, E. U. and Wedley, W. C. (2004). A common framework for deriving preference values from pairwise comparison matrices. Computers $\&$ Operations Research, 31(6):893-908.

Clarke, S. R. and Dyte, D. (2000). Using official ratings to simulate major tennis tournaments. International Transactions in Operational Research, 7(6):585-594.

Crawford, G. and Williams, C. (1985). A note on the analysis of subjective judgment matrices. Journal of Mathematical Psychology, 29(4):387-405.

Csató, L. (2013). Ranking by pairwise comparisons for Swiss-system tournaments. Central European Journal of Operations Research, 21(4):783-803.

Dahl, G. (2012). A matrix-based ranking method with application to tennis. Linear Algebra and its Applications, 437(1):26-36.

De Graan, J. G. (1980). Extensions of the multiple criteria analysis method of T. L. Saaty. Voorburg. National Institute for Water Supply.

Dingle, N., Knottenbelt, W., and Spanias, D. (2013). On the (Page) Ranking of professional tennis players. In Tribastone, M. and Gilmore, S., editors, Computer Performance Engineering, Lecture Notes in Computer Science, pages 237-247. Springer Berlin Heidelberg.

Fedrizzi, M. and Giove, S. (2007). Incomplete pairwise comparison and consistency optimization. European Journal of Operational Research, 183(1):303-313.

Harker, P. T. (1987). Incomplete pairwise comparisons in the analytic hierarchy process. Mathematical Modelling, 9(11):837-848.

Irons, D. J., Buckley, S., and Paulden, T. (2014). Developing an improved tennis ranking system. Journal of Quantitative Analysis in Sports, 10(2):109-118. 
Kéri, G. (2011). On qualitatively consistent, transitive and contradictory judgment matrices emerging from multiattribute decision procedures. Central European Journal of Operations Research, 19(2):215-224.

Kwiesielewicz, M. (1996). The logarithmic least squares and the generalized pseudoinverse in estimating ratios. European Journal of Operational Research, 93(3):611-619.

Kwiesielewicz, M. and van Uden, E. (2003). Ranking decision variants by subjective paired comparisons in cases with incomplete data. In Kumar, V., Gavrilova, M. L., Tan, C. J. K., and L'Ecuyer, P., editors, Computational Science and Its Applications - ICCSA 2003, volume 2669 of Lecture Notes in Computer Science, pages 208-215. Springer, Berlin Heidelberg.

Lin, Y.-K. (2007). On a multicommodity stochastic-flow network with unreliable nodes subject to budget constraint. European Journal of Operational Research, 176(1):347-360.

McHale, I. and Morton, A. (2011). A Bradley-Terry type model for forecasting tennis match results. International Journal of Forecasting, 27(2):619-630.

Motegi, S. and Masuda, N. (2012). A network-based dynamical ranking system for competitive sports. Scientific reports, 2.

Rabinowitz, G. (1976). Some comments on measuring world influence. Conflict Management and Peace Science, 2(1):49-55.

Radicchi, F. (2011). Who is the best player ever? A complex network analysis of the history of professional tennis. PloS one, 6(2):e17249.

Ruiz, J. L., Pastor, D., and Pastor, J. T. (2013). Assessing professional tennis players using Data Envelopment Analysis (DEA). Journal of Sports Economics, 14(3):276-302.

Saaty, T. L. (1980). The Analytic Hierarchy Process: planning, priority setting, resource allocation. McGraw-Hill, New York.

Shiraishi, S. and Obata, T. (2002). On a maximization problem arising from a positive reciprocal matrix in ahp. Bulletin of informatics and cybernetics, 34(2):91-96.

Shiraishi, S., Obata, T., and Daigo, M. (1998). Properties of a positive reciprocal matrix and their application to AHP. Journal of the Operations Research Society of Japan-Keiei Kagaku, 41(3):404-414.

Spanias, D. and Knottenbelt, W. (2013). Tennis player ranking using quantitative models. Manuscript.

http://www.doc.ic.ac.uk/ wjk/publications/spanias-knottenbelt-mis-2013.pdf.

Takeda, E. and Yu, P.-L. (1995). Assessing priority weights from subsets of pairwise comparisons in multiple criteria optimization problems. European Journal of Operational Research, $86(2): 315-331$.

Temesi, J., Csató, L., and Bozóki, S. (2012). Mai és régi idők tenisze - A nem teljesen kitöltött páros összehasonlítás mátrixok egy alkalmazása. In Solymosi, T. and Temesi, J., editors, Egyensúly és optimum. Tanulmányok Forgó Ferenc 70. születésnapjára, pages 213-245. Aula Kiadó, Budapest. 